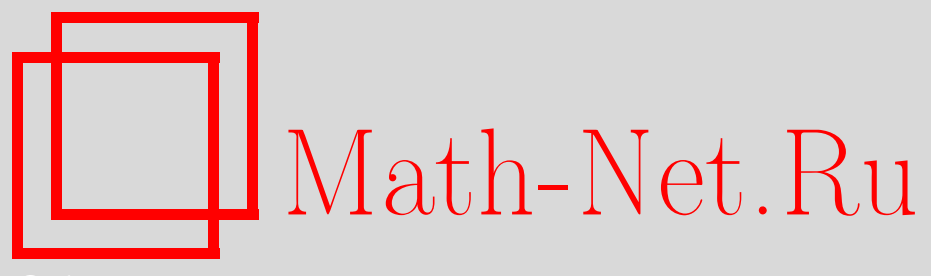

С. С. Синельников, О совместном распределении $(\sup X-X, \sup X)$ для процесса Леви $X, У M H, 2010$, том 65, выпуск 6, 193-194

DOI: https://doi.org/10.4213/rm9395

Использование Общероссийского математического портала Math-Net.Ru подразумевает, что вы прочитали и согласны с пользовательским соглашением http://www.mathnet.ru/rus/agreement

Параметры загрузки:

IP: 3.85 .5 .30

26 апреля 2023 г., 15:00:54

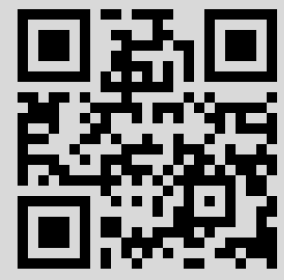




\section{О совместном распределении $(\sup X-X, \sup X)$ для процесса Леви $X$ С. С. Синельников}

1. Классический результат П. Леви гласит (см., например, [1]), что

$$
(\sup B-B, \sup B) \stackrel{\text { law }}{=}(|B|, L(B))
$$

где $B$ - стандартное броуновское движение, $L(B)$ - локальное время броуновского движения в нуле, $\mathrm{a} \stackrel{\text { law }}{=}$ означает совпадение процессов по распределению.

Этот результат обобщается на случай броуновского движения со сносом $B^{\mu}$. В работе [2] (см. также [3]) было показано, что

$$
\left(\sup B^{\mu}-B^{\mu}, \sup B^{\mu}\right) \stackrel{\text { law }}{=}\left(\left|X^{\mu}\right|, L\left(X^{\mu}\right)\right),
$$

где $X^{\mu}$ - единственное решение (сильное) стохастического дифференциального уравнения

$$
d X_{t}^{\mu}=-\mu \operatorname{sgn} X_{t}^{\mu} d t+d B_{t}, \quad X_{0}^{\mu}=0,
$$

где $\operatorname{sgn} x=1$, если $x>0$, и $\operatorname{sgn} x=-1$, если $x \leqslant 0$. Пусть $X_{t}-$ некоторый процесс Леви, мера Леви $\nu$ которого удовлетворяет условию $\int_{|x|<1} \nu(d x)<\infty$. В этом случае число скачков процесса на любом конечном интервале конечно, и для процесса $X_{t}$ можно определить триплет инфинитезимальных характеристик $(\mu, \sigma, \nu)$ относительно функции урезания $h(x)=x \mathscr{I}\{x \neq 0\}$, где $\mathscr{I}$ обозначает индикаторную функцию. Предположим также, что $\sigma>0$, т. е. процесс $X_{t}$ имеет невырожденную диффузионную компоненту. Пусть $S_{t}=\sup _{u \leqslant t} X_{u}$.

Основной результат настоящей работы состоит в следующем.

Теорема. 1) Предположим, что процесс $X_{t}$ не имеет положительных скачков. Тогда пара прочессов $\left(S_{t}-X_{t}, S_{t}\right)$ совпадает по распределению с парой процессов $\left(\left|Y_{t}\right|, L_{t}\right)$, где $Y_{t}$ является решением стохастического дифференциалъного уравнения

$$
d Y_{t}=-\mu \operatorname{sgn} Y_{t-} d t+\sigma d B_{t}-\operatorname{sgn} Y_{t-} \Delta N_{t}, \quad Y_{0}=0,
$$

где $B_{t}$ - стандартное броуновское движение, $N_{t}$ - составной пуассоновский процесс, соответствующий случайной пуассоновской мере интенсивности $\nu, \Delta N_{t}=$ $N_{t}-N_{t-}$, а $L_{t}-$ локальное время марковского прочесса $Y_{t}$ в нуле.

2) В общем случае, когда прочесс $X_{t}$ имеет как положительные, так и отриц,ательные скачки, пара процессов $\left(S_{t}-X_{t}, S_{t}\right)$ совпадает по распределению с парой прочессов $\left(\left|Z_{t}\right|, R_{t}\right)$, где $Z_{t}$ является решением уравнения

$$
\begin{aligned}
d Z_{t}=- & \mu \operatorname{sgn} Z_{t-} d t+\sigma d B_{t}-\operatorname{sgn} Z_{t-} \Delta N_{t} \\
& \quad-\mathscr{I}\left\{Z_{t-}\left(Z_{t-}-\operatorname{sgn} Z_{t-} \Delta N_{t}\right) \leqslant 0\right\}\left(Z_{t-}-\operatorname{sgn} Z_{t-} \Delta N_{t}\right), \quad Z_{0}=0,
\end{aligned}
$$

где $B_{t}$ и $N_{t}$ были определенъ ранее,

$$
R_{t}=\sum_{0 \leqslant s \leqslant t} \mathscr{I}\left\{Z_{s-}\left(Z_{s-}-\operatorname{sgn} Z_{s-} \Delta N_{s}\right) \leqslant 0\right\}\left|Z_{s-}-\operatorname{sgn} Z_{s-} \Delta N_{s}\right|+L_{t},
$$

a $L_{t}$ является локальным временем марковского процесса $Z_{t}$ в нуле. 
2. Докажем теорему в общем случае. Рассмотрим процесс $Y_{t}$, удовлетворяющий уравнению

$$
d Y_{t}=-\mu \operatorname{sgn} Y_{t-} d t+\sigma d B_{t}-\operatorname{sgn} Y_{t-} \Delta N_{t}, \quad Y_{0}=0 .
$$

Поскольку процесс $N_{t}$ имеет конечное число скачков на любом конечном интервале, то, используя результаты работы [4], можно показать, что это уравнение имеет единственное сильное решение, к тому же являющееся строго марковским процессом. Процесс $Z_{t}$ может быть восстановлен из процесса $Y_{t}$ с помощью техники замещения:

$$
Z_{t}= \begin{cases}Y_{t}^{1}=Y_{t}, & 0 \leqslant t<\tau_{1}, \\ Y_{t}^{2}=Y_{t}-Y_{\tau_{1}}^{1}, & \tau_{1} \leqslant t<\tau_{2}, \\ Y_{t}^{3}=Y_{t}-Y_{\tau_{1}}^{1}-Y_{\tau_{2}}^{2}, & \tau_{2} \leqslant t<\tau_{3}, \\ \ldots, & \end{cases}
$$

где $\tau_{i}$ - моменты, когда процесс $Y_{t}^{i}$ пересекает ось абсцисс скачком. Таким образом, процесс $Z_{t}$, определенный как решение уравнения (1), существует, единственен и является строго марковским процессом. Заметим, что из представления (2) следует, что все скачки процесса $Z_{t}$ остаются в одной полуплоскости (дополненной нулем).

Применим к процессу $\left|Z_{t}\right|$ формулу Танака:

$$
\left|Z_{t}\right|=\int_{0}^{t} \operatorname{sgn} Z_{s-} d Z_{s}+\sum_{0 \leqslant s \leqslant t}\left\{\left|Z_{s}\right|-\left|Z_{s-}\right|-\operatorname{sgn} Z_{s-} \Delta Z_{s}\right\}+L_{t},
$$

где $L_{t}$ - локальное время процесса $Z_{t}$ в нуле. В уравнении (3) второй член отвечает за компенсацию тех скачков процесса $Z_{t}$, при которых процесс пересекает нулевой уровень. Поскольку в процессе $Z_{t}$ таких скачков нет, то этот член равен нулю. Подставляя $d Z_{s}$ из уравнения (1) в уравнение (3) и обозначая $P_{t}=\mu \int_{0}^{t} d s-\int_{0}^{t} \operatorname{sgn} Z_{s-} d B_{s}+$ $\sum_{0 \leqslant s \leqslant t} \Delta N_{s}$, получаем

$\left|Z_{t}\right|=-P_{t}+\sum_{0 \leqslant s \leqslant t} \mathscr{I}\left\{Z_{s-}\left(Z_{s-}-\operatorname{sgn} Z_{s-} \Delta N_{s}\right) \leqslant 0\right\}\left|Z_{s-}+\left(Z_{s-}-\operatorname{sgn} Z_{s-} \Delta N_{s}\right)\right|+L_{t}$.

Заметим, что $\int_{0}^{\infty} \mathscr{I}\left\{\left|Z_{t}\right| \neq 0\right\} d R_{t}=0$, где $R_{t}$ обозначает сумму двух последних слагаемых в (4). Тогда к уравнению (4) можно применить лемму Скорохода для càdlàg функций (см., например, [5]). Учитывая, что $P_{0}=0$, мы приходим к выражению

$$
\left(\sup _{s \leqslant t} P_{s}-P_{t}, \sup _{s \leqslant t} P_{s}\right)=\left(\left|Z_{t}\right|, R_{t}\right) \quad \forall t \geqslant 0 .
$$

Из характеризационной теоремы Леви следует, что $P_{t} \stackrel{\text { law }}{=} X_{t}$. Таким образом, из (5) вытекает искомое утверждение.

\section{Список литературы}

[1] П. Леви, Стохастические прочессы и броуновское движение, Наука, М., 1972, 375 с. [2] S. E. Graversen, A. N. Shiryaev, Bernoulli, 6:4 (2000), 615-620. [3] А. С. Черный, А. Н. Ширяев, Теория вероятн. и ее примен., 44:2 (1999), 466-472. [4] А. К. Звонкин, Матем. сб., 93:1 (1974), 129-149. [5] H. J. Engelbert, V. Kurenok, A. Zalinescu, From stochastic calculus to mathematical finance, Springer, Berlin, 2006, 227-249.

C. C. Синельников (S.S. Sinelnikov)

Московский государственный университет им. М. В. Ломоносова

E-mail: sergesinelnikov@gmail.com
Представлено Д. В. Трещёвым Принято редколлегией 09.10 .2010 